\title{
On the Theory of Topological Computation in the Lowest Landau Level of QHE
}

\author{
Dipti Banerjee \\ Department of Physics, Vidyasagar College for Women, Kolkata, India \\ E-mail: deepbancu@homail.com,dbancu@gmail.com \\ Received August 28, 2011; revised November 20, 2011; accepted November 28, 2011
}

\begin{abstract}
We have studied the formation of Hall-qubit in lowest Landau level of (LLL) Quantum Hall effect due to the Aharonov-Bohm oscillation of quasiparticles. The spin echo method plays the key role in the topological entanglement of qubits. The proper ratio of fluxes for maximally entangling qubits has also been pointed out. The generation of higher Quantum Hall state may be possible with the help of quantum teleportation.
\end{abstract}

Keywords: Spin Echo, Aharonov-Bohm Phase

\section{Introduction}

Entanglement is one of the basic aspects of Quantum mechanics that exhibits the peculiar correlations between two physically distant parts of the total systems. The Geometric phase such as Berry phase (BP) [1] and Aharonov-Bohm phase [2] play an important role in Quantum mechanics. The effect of Berry phase on the entangled quantum system is less known. The quantum gates that convey topological transformation is known as topological gates. These gates are advantageous for their immunity and resistive power for local disturbances. They do not depend on the overall time evolution nor on small deformations on the control parameter. This indicates that a quantum mechanical state would carry its memory during its spatial variation and the influence of Berry phase on an entangled state could be linked up with the local observations of spins. Few attempts have been made connecting the Berry phase with entanglement of spin- $1 / 2$ particles resulting the outcome of Geometric/Holonomic quantum computation [3].

The transport of information through quasi-particles exhibiting long-range non-abelian Aharonov-Bohm interactions can yield similar topological quantum computation [4]. Kitaev [5] pointed out topological quantum computer as a device in which quantum numbers carried by quasi-particles residing on 2DES have long range Aharonov-Bohm interactions with one another. There exist a strong quantum correlation between the quasiparticles interacting by A-B effect extending out over large distances [6].
The interwinding of the quasi-particles trajectories in the course of time evolution of the qubits realizes controlled-phase transformations with nontrivial phase values. One of the remarkable discoveries of recent decades is the infinite range A-B interactions observed in Fractional Quantum Hall effect [7]. The electrons in the Quantum Hall systems are so highly frustrated that the ground state is an extremely entangled state. The quantum entanglement of particle having nontrivial Berry phase is associated with the transport of a charge around a flux which is equivalent to the Aharonov-Bohm phase in the analogy with the gauge theory. A similar reflection is seen in FQHE when one quasi-particle/composite particle goes around another encircling an area $\mathrm{A}$. The total phase associated with this path is given by [8]

$$
\Phi^{*}=-2 \pi\left(\mathrm{BA} / \phi_{0}-2 p N_{e n c}\right)
$$

where $N_{e n c}$ is the number of composite fermion inside the loop. The first term on the right hand side is usual A$\mathrm{B}$ phase and the second term is the contribution from the vortices bound to composite fermions indicating that each enclosed composite fermion effectively reduces the flux by $2 p$ flux quanta. These particles of a nontrivial condensed matter state obey fractional statistics and Arovas et al. [9] pointed out that the exchange of particles over a half loop producing phase factor $e^{i \pi \theta}=(-1)^{\theta}$.

In the entanglement of two spin- $1 / 2$ particles in presence of magnetic field through the spin echo method BP plays an important role [10]. We have studied in presence of Rabi oscillation the rotation of qubit through BP 
[11]. A reflection of this idea is seen to study the formation of Hall qubit rotation recently in $v=1$ the ground state in the light of quantum entanglement [12]. Here we aim at understanding the rotation of Hall qubits having A-B interactions in the background of topological computation.

\section{Formation of Hall-Qubit from Berry Phase}

One single qubit can be sufficiently constructed [3] using the two well known quantum gates-Hadamard gate $(\mathrm{H})$ and Phase gate as follows

$$
\begin{aligned}
& |0\rangle--[H]--\bullet^{2 \theta}--[H]--\bullet^{\pi / 2+\phi} \\
& \rightarrow \cos \theta|0\rangle+\sin \theta e^{i \phi}|1\rangle
\end{aligned}
$$

With the use of quantum gates and elementary qubits $|0\rangle$ and $|1\rangle$ the spinors for up or down polarization can be written as

$$
|\uparrow\rangle=\left(\sin \frac{\theta}{2} e^{i \phi / 2}|0\rangle+\cos \frac{\theta}{2}|1\rangle\right)
$$

and

$$
|\downarrow\rangle=\left(-\cos \frac{\theta}{2}|0\rangle+\sin \frac{\theta}{2} e^{i \phi / 2}|1\rangle\right)
$$

This above qubit representing a spinor acquires the geometric phase [11] over a closed path.

$$
\begin{aligned}
\gamma_{\uparrow} & =i \oint\langle\uparrow, t|\nabla| \uparrow, t\rangle \cdot \mathrm{d} \lambda \\
& =i \oint A_{\uparrow}(\lambda) \mathrm{d} \lambda \\
& =\oint L_{\text {eff }} \mathrm{d} t \\
& =-\pi(1-\cos \theta)
\end{aligned}
$$

which is a solid angle subtended about the quantization axis. For the conjugate state the Berry phase over the closed path becomes

$$
\gamma_{\downarrow}=\pi(1-\cos \theta)
$$

Here the angle $\theta$ measures the deviation of the magnetic flux line from the Z-axis. The fermionic or the antifermionic nature of the two spinors (up/down) can be identified by the maximum value of topological phase $\gamma_{\uparrow / \downarrow}= \pm \pi$ at an angle $\theta=\pi / 2$. For $\theta=0$ we get the minimum value of $\gamma_{\uparrow}=0$ and at $\theta=\pi$ no extra effect of phase is realized.

In the spin echo method, this Berry phase can be fruitfully $[13,14]$ isolated in the construction of two qubit through rotation of one qubit (spin 1/2) in the vicinity of another. Incorporating the spin-echo for half period we find the antisymmetric Bell's state after one cycle $(t=\tau)$,

$$
\left|\Psi_{-}(t=\tau)\right\rangle=\frac{1}{\sqrt{2}}\left(\mathrm{e}^{i \gamma \uparrow}|\uparrow\rangle_{1} \otimes|\downarrow\rangle_{2}-\mathrm{e}^{-i \gamma \uparrow}|\downarrow\rangle_{1} \otimes|\uparrow\rangle_{2}\right)
$$

And symmetric state becomes

$$
\left|\Psi_{+}(t=\tau)\right\rangle=\frac{1}{\sqrt{2}}\left(\mathrm{e}^{-i \gamma \uparrow}|\uparrow\rangle_{1} \otimes|\downarrow\rangle_{2}+\mathrm{e}^{i \gamma \uparrow}|\downarrow\rangle_{1} \otimes|\uparrow\rangle_{2}\right)
$$

where $\gamma_{\downarrow}=-\gamma_{\uparrow}=-\gamma$. It may be noted that for $\left|\mu_{\uparrow}\right|=\frac{1}{2}(1-\cos \theta)$.

This phase plays the key role through the $\mu$ factor, in the measurement of entanglement by the concurrence "C" of an entangled state. For a two qubit state $|\psi\rangle=\beta|\uparrow \downarrow\rangle+\gamma|\downarrow \uparrow\rangle$ the concurrence [4] is given by

$$
C=2 \beta \gamma
$$

When $C=1$ the entanglement is maximum and disentanglement for $C=0$. The value of $\left|\mu_{\uparrow}\right|=0$ implies disentanglement for $\theta=0$. For $\theta=\pi$ there is maximum deviation of flux line yielding $\left|\mu_{\uparrow}\right|=1$ as a signature of maximum entanglement.

Splitting up these above two Equations (11) and (12) into the symmetric and antisymmetric states [12] and rearranging we have

$$
\begin{aligned}
& \left|\Psi_{+}\right\rangle_{\tau}=\cos \gamma\left|\Psi_{+}\right\rangle_{0}-i \sin \gamma\left|\Psi_{-}\right\rangle_{0} \\
& \left|\Psi_{-}\right\rangle_{\tau}=i \sin \gamma\left|\Psi_{+}\right\rangle_{0}+\cos \gamma\left|\Psi_{-}\right\rangle_{0}
\end{aligned}
$$

The doublet acquiring the matrix Berry phase $\Sigma$ as rotated from $t=0$ to $t=\tau$.

$$
\begin{gathered}
\left(\begin{array}{l}
\left|\Psi_{+}\right\rangle \\
\left|\Psi_{-}\right\rangle
\end{array}\right)_{\tau}=\sum\left(\begin{array}{l}
\left|\Psi_{+}\right\rangle \\
\left|\Psi_{-}\right\rangle
\end{array}\right)_{0} \\
\Sigma=\left(\begin{array}{cc}
\cos \gamma & -i \sin \gamma \\
i \sin \gamma & \cos \gamma
\end{array}\right)=\cos 2 \gamma
\end{gathered}
$$

This non-abelian matrix Berry phase $\Sigma$ is developed from the abelian Berry phase $\gamma$. For $\gamma=0$ there is symmetric rotation of states, but for $\gamma=\pi$ the return is antisymmetric and the respective values of $\Sigma=$ I and $-\mathrm{I}$ (where I = identity matrix).

There is a deep analogy between FQHE and superfluidity [15]. The ground state of anti-ferromagnetic Heisenberg model on a lattice introduce frustration giving rise to the resonating valence bond (RVB) states corresponding spin singlets where two nearest-neighbor bonds are allowed to resonate among themselves. The RVB state is a coherent superposition of spin singlet pairs and 
can be written as

$$
|\mathrm{RVB}\rangle=\sum\left(i_{1} j_{1}, \cdots, i_{n} j_{n}\right) \prod\left(i_{k} j_{k}\right)
$$

in which $(i, j)=\frac{1}{\sqrt{2}}(i \uparrow j \downarrow-i \downarrow j \uparrow)$ is a spin singlet pair (valance bond) between sites $i$ and $j$. This RVB state support fractionalized excitation of spin $1 / 2$ spinon $[4,5]$. The topological order is closely related to the coherent motion of fractionalised spin excitation in RVB background. It is suggested that RVB states [6] is a basis of fault tolerant topological quantum computation. Since these spin singlet states forming a RVB gas is equivalent to fractional quantum Hall fluid, its description through quantum computation will be of ample interest.

The Quantum Hall effect can be considered on a 3D anisotropic space having the N-particle wave-function of parent Hall states

$$
\Psi_{N_{\uparrow}}^{(m)}=\prod\left(u_{i} v_{j}-u_{j} v_{i}\right)^{m}
$$

Represent the one qubit in the language of quantum information. In the Jain's formalism [16], $\Psi_{v}^{m}$ the hierarchical FQHE incompressible state for Landau filling factor $v=\frac{p}{q}=\frac{n}{n(m-1) \pm 1}=\frac{2 m n \pm 1}{n}$ becomes

$$
\left|\Psi_{v}(z)^{m}\right\rangle=\left|\Phi_{n}(z)\right\rangle\left|\Phi_{1}(z)\right\rangle^{m-1}=\left|\Phi_{1}(z)\right\rangle^{2 m+1 / n}
$$

where $m=$ odd for making the state $\Psi_{v}(z)^{m}$ antisymmetric and $n=$ integer, specifying Lanadu level in QHE. The state $\Phi_{1}(z)$ is the QHE state at the lowest landau level $n=1$ and filling factor $v=1$. States of the above form are grouped into a family depending on the values of $m$. Any FQHE state can be expressed in terms of the IQHE ground state [17].

Recently we have identified [12] this ground state $\Phi_{1}(z)$ as the Hall qubit, the basic building block for constructing any other IQHE/FQHE state formed when two nearest neighbor bonds are allowed to resonate among themselves. It is an extremely entangled state visualized by the formation of singlet state between a pair of $(i, j)^{\text {th }}$ spinors.

$$
\begin{aligned}
\Phi_{1}(z) & =\left(u_{i} v_{j}-u_{j} v_{i}\right)=\left(\begin{array}{ll}
u_{i} & v_{i}
\end{array}\right)\left(\begin{array}{cc}
0 & 1 \\
-1 & 0
\end{array}\right)\left(\begin{array}{l}
u_{j} \\
v_{j}
\end{array}\right) \\
& =\left\langle\uparrow_{i}\left|\left(\begin{array}{cc}
0 & 1 \\
-1 & 0
\end{array}\right)\right| \uparrow_{j}\right\rangle
\end{aligned}
$$

A singlet state is a two qubit developed as one qubit $|\uparrow\rangle=\left(\begin{array}{l}u \\ v\end{array}\right)=\left(\begin{array}{c}\sin \frac{\theta}{2} \mathrm{e}^{i \phi} \\ \cos \frac{\theta}{2}\end{array}\right)$ rotates in the vicinity of another through the spin echo method where Berry's topological phase dominates in acquiring the Hall qubit with the description of a two component up spinor.

In this present work we have focused on Quantum Hall effect where the nature of the state will be only antisymmetric. Hence the Equation (17) reduces to

$$
\left|\Psi_{-}\right\rangle_{\tau}=\sum\left|\Psi_{-}\right\rangle_{0}
$$

The Hall qubit $\Phi_{1}(z)$ has resemblance with $\left|\Psi_{-}\right\rangle$. In the lowest Landau level $v=m=1$ would develop similar non-abelian Berry phase $\Sigma$. This is visualizing the spin conflict during parallel transport leading to matrix Berry phase. Over a closed period $t=\tau$ the QHE state $\Phi_{1}(z)$ at $v=1$ filling factor will acquire the matrix Berry phase.

$$
\left|\Phi_{1}(z)\right\rangle_{\tau}=\mathrm{e}^{i \gamma_{\uparrow}^{H}}\left|\Phi_{1}(z)\right\rangle_{0}
$$

Here $\mathrm{e}^{i \gamma^{H}}$ is the non-abelian matrix Berry phase

$$
\gamma_{\uparrow}^{H}=\left(\begin{array}{ll}
\gamma_{i} & \gamma_{i j} \\
\gamma_{j i} & \gamma_{j}
\end{array}\right)
$$

where $\gamma_{i}$ and $\gamma_{j}$ are the BPs for the ith and jth spinor as seen in Equation (16) and the off-diagonal BP $\gamma_{i j}$ arises due to local frustration in the spin system.

All the above explanation is restricted for lowest Landau level $v=1$, but concentrating on the other parent state $v=1 / m$ where $m=$ odd integers, the Berry phase of a qubit is $\gamma=i m \pi$ [12] that is associated with the two qubit Hall state

$$
\left|\Phi_{1}(z)\right\rangle=\frac{1}{\sqrt{2}}\left(|\uparrow\rangle_{1}|\downarrow\rangle_{1}\right)\left(\begin{array}{cc}
0 & -\mathrm{e}^{-i m \pi} \\
\mathrm{e}^{i m \pi} & 0
\end{array}\right)\left(\begin{array}{l}
|\uparrow\rangle_{2} \\
|\downarrow\rangle_{2}
\end{array}\right)
$$

through the process of quantum entanglement between two one qubit where the reflection of spin echo is visualized.

\section{Hall-Qubit Formation in QHE through Aharonov-Bohm Phase}

In the composite fermion theory of Quantum Hall effect the qubits are equivalent to the fluxes attached with the charged particles. When an electron is attached with a magnetic flux, its statistics changes and it is transformed into a boson. These bosons condense to form cluster which is coupled with the residual fermion or boson (composed of two fermions). Indeed the residual boson or fermion will undergo a statistical interaction tied to a geometric Berry phase effect that winds the phase of the particles as it encircles the vortices. Indeed as two vortices cannot be brought very close to each other, there will be a hard core repulsion in the system which ac- 
counts for the incompressibility of the Quantum Hall fluid.

These non-commuting fluxes have their own interesting Aharonov-Bohm interactions. As the quasi-particles encircle another in their way of topological transport, the Aharonov-Bohm type statistical phase is developed. Following a generalization of Pauli exclusion principle, Haldane [18] pointed out that the quasi-particles carrying flux $\phi_{\alpha}$ and charge $q_{\beta}$ orbiting around another object carrying flux $\phi_{\beta}$ and charge $q_{\beta}$ has the relative statistical phase $\theta_{\alpha \beta}$

$$
\exp \left(i \theta_{\alpha \beta}\right)=\exp \pm i \pi\left(g_{\alpha \beta}+g_{\beta \alpha}\right)
$$

where $g_{\alpha \beta}=-q_{\alpha} \phi_{\beta}$. With this view we have recently shown that when two non-identical composite fermions residing in two consecutive Landau levels in FQHE encircle each other, the relative Aharonov-Bohm (AB) type phase is developed. As the quasi-particles advance towards the edge of FQHE in a similar circular way, the developed current [19] should have a connection with this $\mathrm{AB}$ type phase through Berry's topological phase.

These A-B interactions are the key source of forming two qubit Hall states identified as Hall qubit. Hence movement of Hall qubits would develop the A-B phase. In the physics of spin echo instead of Berry phase the incorporation of Aharonov-Bohm phase would be more appropriate as the rotation of qubits are equivalent to the rotation of fluxes around charges. If $\mathrm{e}^{i \phi s}$ be the Aharonov-Bohm phase between the two qubits, for half period we find the antisymmetric Bell's state after one cycle $(t=\tau)$,

$$
\left|\Psi_{-}(t=\tau)\right\rangle=\frac{1}{\sqrt{2}}\left(\mathrm{e}^{i \phi s}|\uparrow\rangle_{1} \otimes|\downarrow\rangle_{2}-\mathrm{e}^{i \phi s}|\downarrow\rangle_{1} \otimes|\uparrow\rangle_{2}\right)
$$

Similar consideration for symmetric states

$$
\left|\Psi_{+}(t=\tau)\right\rangle=\frac{1}{\sqrt{2}}\left(\mathrm{e}^{\mathrm{i} \phi s}|\uparrow\rangle_{1} \otimes|\downarrow\rangle_{2}+\mathrm{e}^{i \phi s}|\downarrow\rangle_{1} \otimes|\uparrow\rangle_{2}\right)
$$

Splitting up these states and rearranging the symmetric and antisymmetric parts we have the doublet acquiring the matrix form of Aharonov-Bohm phase $\Upsilon$ as rotated from $t=0$ to $t=\tau$.

$$
\left(\begin{array}{l}
\left|\Psi_{+}\right\rangle \\
\left|\Psi_{-}\right\rangle
\end{array}\right)_{\tau}=\Upsilon\left(\begin{array}{l}
\left|\Psi_{+}\right\rangle \\
\left|\Psi_{-}\right\rangle
\end{array}\right)_{0}
$$

where

$$
\Upsilon=\left(\begin{array}{cc}
\cos \phi_{s} & -i \sin \phi_{s} \\
i \sin \phi_{s} & \cos \phi_{s}
\end{array}\right)=\cos 2 \phi_{s}
$$

This topological matrix phase $\Upsilon$ is developed from the Aharonov-Bohm phase $\phi_{s}$ as one qubit rotates around another. The qubits in QHE are quantized spinor having flux attached with charge. Their entanglement is equivalent to spin type echo where the topological phase dominates due to Aharonov-Bohm oscillation between them. This compel to change the Berry phase of the singlet state as in Equation (24) by the relative A-B type phase $\phi_{s}$.

$$
\left|\Phi_{1}(z)\right\rangle_{\tau}=\mathrm{e}^{\mathrm{i} \phi s}\left|\Phi_{1}(z)\right\rangle_{0}
$$

This Hall qubit can be visualized in terms of entanglement of two oscillating qubits.

$$
\left|\Phi_{1}(z)\right\rangle=\frac{1}{\sqrt{2}}\left(\begin{array}{ll}
|\uparrow\rangle_{1} & |\downarrow\rangle_{1}
\end{array}\right)\left(\begin{array}{cc}
0 & -\mathrm{e}^{i \phi s} \\
\mathrm{e}^{i \phi s} & 0
\end{array}\right)\left(\begin{array}{l}
|\uparrow\rangle_{2} \\
|\downarrow\rangle_{2}
\end{array}\right)
$$

To form the singlet state between the qubits under A-B interactions in the spin echo method, the essential condition for antisymmetric QHE states are visualized by $\pm \mathrm{e}^{i \phi s}= \pm \mathrm{e}^{i \pi}= \pm 1$.

In the formation of Hall qubit through the entangling of qubits in the different Landau level the A-B phase plays the key role in the spin type echo method. If the rotating qubits are in the same Landau level, the A-B phase changes to statistical phase. Considering the interaction [20] between two identical qubits in the same $n^{\text {th }}$ Landau level for the composite particles filling factor $v=\frac{n}{2 \mu_{\text {eff }}}$ the statistical phase becomes

$$
\phi_{s}=\exp \pm i \frac{n \pi}{2}
$$

where for $n=2,4,6 \cdots$ the change of statistics will be fermionic visualized by the phase $\phi_{s}=\exp \pm i \pi$. On the other hand for $n=1,3,5 \cdots$ the statistics will be bosonic $\phi_{s}=\exp \pm i \pi / 2$. It may be noted that for fractional filling factors the final statistics will be fermionic through proper combinations. With this view, the entanglement of two one qubits in the same Landau Level, the Hall qubit $\left|\Phi_{1}(z)\right\rangle$ will be formed when the exchange phase is $\pm i \pi$

$$
\left|\Phi_{1}(z)\right\rangle=\frac{1}{\sqrt{2}}\left(|\uparrow\rangle_{1} \quad|\downarrow\rangle_{1}\right)\left(\begin{array}{cc}
0 & -\mathrm{e}^{-i \pi} \\
\mathrm{e}^{-i \pi} & 0
\end{array}\right)\left(\begin{array}{l}
|\uparrow\rangle_{2} \\
|\downarrow\rangle_{2}
\end{array}\right)
$$

Whenever the interaction takes place between dissimilar qubits in different Landau level the rotation of one against another develops the Aharonov-Bohm type phase that does not express their statistics. We assumed the transfer of the composite particle [19] from the inner edge in the $n^{\text {th }}$ Landau level having filling factor $v_{n}$ picking up even integral $(2 \mathrm{~m})$ of flux $v_{1}$ through the bulk of QH system and forming a new composite particle in the $(n+1)^{\text {th }}$ Landau level in the outer edge. The fill- 
ing factor of the effective particle becomes $v_{\text {eff }}=\frac{n+1}{\mu_{\text {eff }}}$. The monopole strength $\mu_{\text {eff }}$ of the state $\Phi_{1}^{2 m} \Phi_{n}$ can be considered as

$$
\mu_{\text {eff }}=2 m \mu_{1}+\mu_{n} .
$$

Encircling of the composite particle in the inner edge having flux $\mu_{n}$ with charge $q_{n}$ around the composite particle in the outer edge having corresponding flux $\mu_{\text {eff }}$ would develop a relative $\mathrm{AB}$ type phase

$$
\phi_{s}=\exp \pm \frac{i \pi}{2}\left(q_{n} \mu_{\text {eff }}+q_{\text {eff }} \mu_{n}\right)
$$

In more simplified way it becomes

$$
\phi_{s} \cong \exp \pm \frac{i \pi}{2}\left[\left(n+\frac{1}{2}\right)-m \frac{\mu_{1}}{\mu_{n}}\right]
$$

Since the concurrence $C=1$ indicate the maximum entanglement and for disentanglement the value of minimum concurrence is $C=0$, we can establish a relationship between the fluxes of the entangling qubits on the Hall surface. The maximum entanglement between the two quasi-particle results a relation between the respective two fluxes $\mu_{1}$ and $\mu_{n}$ in terms of parent filling factor $m$ and Landau level $n$.

$$
\exp \pm \frac{i \pi}{2}\left[\left(n+\frac{1}{2}\right)-m \frac{\mu_{1}}{\mu_{n}}\right]=\exp \pm i \pi
$$

This gives a ratio between the entangling fluxes $\mu_{n}$ and $\mu_{1}$ in order to form the singlet pairs through $\mathrm{AB}$ oscillations in Quantum Hall effect.

$$
\mu_{n}=\frac{2 m}{2 n-3} \mu_{1}
$$

It may be noted that for maximum entanglement $\mu_{1}=1$ results

$$
\mu_{n}=\frac{2 m}{2 n-3}
$$

and for minimum entanglement both $\mu_{1}$ and $\mu_{n}$ becomes zero.

The physics behind the formation of higher Hall states take place through the entanglement of Hall qubits $\left|\Phi_{1}(z)\right\rangle$ in the lowest landau level. Here the A-B phase or statistical phase plays the key role in the process of spin echo with the essential condition $\pm \mathrm{e}^{\mathrm{i} \phi s}= \pm \mathrm{e}^{i \pi}= \pm 1$. The outcome of entanglement of two Hall qubits is

$$
\begin{aligned}
|\alpha(z)\rangle & =\left\langle\Phi_{1}(z)\left|\left(\begin{array}{cc}
0 & -\mathrm{e}^{-i \pi} \\
\mathrm{e}^{i \pi} & 0
\end{array}\right)\right| \Phi_{1}(z)\right\rangle \\
& =\left(\begin{array}{cc}
0 & \Phi_{1}(z) \\
-\Phi_{1}(z) & 0
\end{array}\right)
\end{aligned}
$$

where $\Phi_{1}(z)=\left(u_{i} v_{j}-v_{i} u_{j}\right)$. On the similar manner we realize that the entanglement of two $|\alpha(z)\rangle$ gives rise to the state formed by the square of Hall qubits.

$$
|\gamma(z)\rangle=\left\langle\alpha(z)\left|\left(\begin{array}{cc}
0 & 1 \\
-1 & 0
\end{array}\right)\right| \alpha(z)\right\rangle=\left(\begin{array}{cc}
0 & \Phi_{1}^{2}(z) \\
-\Phi_{1}^{2}(z) & 0
\end{array}\right)
$$

In order to maintain the antisymmetric nature of the Hall state the power of the Hall qubit should be odd. This is possible only when two asymmetric Hall qubits (one even power with another odd power) entangle under topological interactions

$$
\left\langle\gamma(z)\left|\left(\begin{array}{cc}
0 & 1 \\
-1 & 0
\end{array}\right)\right| \alpha(z)\right\rangle=\left(\begin{array}{cc}
0 & \Phi_{1}^{3}(z) \\
-\Phi_{1}^{3}(z) & 0
\end{array}\right)
$$

Forming a Hall state in the parent Landau filling factor $v=m=$ odd .

We like to conclude mentioning that the hierarchical FQHE states are formed through the process of quantum teleportation. If there are three entities defined by 1,2 , and 3 , then transportation of 1 to 3 through 2 will be

$$
\begin{aligned}
|\Psi\rangle_{123} & =\left|\Phi_{1}\right\rangle\left|\Psi_{23}\right\rangle \\
& =\frac{1}{2}\left(1+\sigma^{1} \cdot \sigma^{3}\right)\left|\Psi_{12}\right\rangle\left|\Phi_{3}\right\rangle
\end{aligned}
$$

Similar reflection of quantum teleportation [21] in FQHE motivate us to write

$$
\Psi_{v}^{m}=\Phi_{1}^{2 m} \Phi_{n}=\Phi_{1}^{2 m} \Phi_{1}^{\frac{1}{n}}=\frac{1}{2}\left(1+\sigma^{1} \cdot \sigma^{3}\right) \mid \Phi_{1}^{\frac{1}{n}} \Phi_{1}^{2 m}
$$

A hierarchical FQHE state whose extensive study through the entanglement of Hall qubits maintaining the antisymmetric nature of the exchange phase is to be done in future.

\section{Conclusions}

In this paper we have studied the Physics behind the Hall qubit formed by the entanglement of two qubits where one is rotating in the field of the other with AharonovBohm (AB) phase. The image of spin echo between the entangling composite fermion/qubit has been reflected in the field of Quantum Hall effect. Topological quantum computation has been executed considering the Hall qubit at $v=1$ as a building block for the formation of other higher IQHE/FQHE states at different filling factors. With the condition of concurrence for maximum entanglement, a proper ratio between the fluxes of the entangling qubits has been evaluated. At the end, we have mentioned that the states in hierarchies of FQHE can be studied in the light of quantum teleportation 
whose extensive study will be of ample interest in future.

\section{Acknowledgements}

This work has been partly supported by The Abdus Salam International Center for Theoretical Physics, Trieste, Italy during the visit as Regular associate.

\section{References}

[1] M. V. Berry, "Quantal Phase Factor Accompanying Adiabatic Changes," Proceedings of the Royal Society A, Vol. 392, No. 1802, 1984, pp. 45-57. doi:10.1098/rspa.1984.0023

[2] Y. Aharonov and J. S. Anandan, "Phase Change during a Cyclic Quantum Evolution," Physical Review Letters, Vol. 58, No. 16, 1987, pp. 1593-1596. doi:10.1103/PhysRevLett.58.1593

[3] V. Vedral, "Geometric Phase and Topological Quantum Computation," Physics, Vol. 1, 2002, pp. 1-24.

[4] R. W. Ogburn and J. Preskill, "Lecture Notes in Computer Science," Springer-Verlag, New York, 1999.

[5] A. Y. Kiteav, "Fault Tolarent Quantum Computation by Anyons," Annals of Physics, Vol. 303, No. 1, 2003, pp. 2-30.

[6] H. K. Lo and J. Preskill, "Non-Abelian Vortices and NonAbelian Statistics," Physical Review D, Vol. 48, No. 10, 1993, pp. 4821-4834.

[7] D. V. Averin and V. J. Goldman, "Quantum Computation with Quasiparticles of the Fractional Quantum Hall Effect," Solid State Communications, Vol. 121, No. 1, 2001, pp. 25-28. doi:10.1016/S0038-1098(01)00447-1

[8] A. S. Goldhaber and J. K. Jain, "Characterization of Fractional-Quantum-Hall-Effect Quasiparticles," Physics Letters A, Vol. 199, No. 3-4, 1995, pp. 267-273. doi:10.1016/0375-9601(95)00101-8

[9] D. Arovas, J. R. Schrieffer and F. Wilczek, "Fractional Statistics and Quantum Hall Effect," Physical Review Letters, Vol. 53, No. 7, 1984, pp. 722-723.
doi:10.1103/PhysRevLett.53.722

[10] V. Vedral, "Entanglement in Second Quantization Formalism," Central European Journal of Physics, Vol. 1, No. 2, 2003, pp. 289-306.

[11] D. Banerjee and P. Bandyopadhyay, "Qubit Rotation and Berry Phase," Physica Scripta, Vol. 73, No. 6, 2006, pp. 571-576. doi:10.1088/0031-8949/73/6/008

[12] D. Banerjee, "Qubit Rotation in QHE," Physica Scripta, Vol. 77, No. 6, 2008, pp. 065701-065705. doi:10.1088/0031-8949/77/06/065701

[13] A. Ekert, M. Ericson, V. Vedral, et al., "Geometric Quantum Computation," Journal of Modern Optics, Vol. 47, No. 14-15, 2000, pp. 2501-2513.

[14] R. A. Bertlmann, K. Durstberger, Y. Hasegawa and B. C. Heismayr, "Berry Phase in Entangled Systems: An Experiment with Single Neutrons," Physical Review A, Vol. 69, No. 3, 2004, pp. 032112-032118. doi:10.1103/PhysRevA.69.032112

[15] R. Prange and S. Girvin, "The Quantum Hall Effect," Spinger-Verlag, New York, 1987.

[16] J. K. Jain, "The Theory of Fractional Quantum Hall Effect," Physical Review B, Vol. 41, No. 11, 1990, pp. 7653 7665. doi:10.1103/PhysRevB.41.7653

[17] D. Banerjee, "Topological Aspects of Shift in Hierarchies of the Fractional Quantum Hall Effect," Physical Review B, Vol. 58, No. 8, 1998, pp. 4666-4671.

[18] F. D. M. Haldane, "Fractional Statistics in Arbitrary Dimensions: A Generalization of the Pauli Principle," Physical Review Letters, Vol. 67, No. 8, 1991, pp. 937-940. doi:10.1103/PhysRevLett.67.937

[19] D. Banerjee, "Edge Current of FQHE and AharonovBohm Type Phase," Physica Scripta, Vol. 71, No. 6, 2005, pp. 656-660. doi:10.1088/0031-8949/71/6/014

[20] D. Banerjee, "Topological Aspects of Phases in Fractional Quantum Hall Effect," Physics Letters A, Vol. 269, No. 2-3, 2000, pp. 138-143. doi:10.1016/S0375-9601(00)00251-6

[21] S. Stenholm and K. Suominen, "Quantum Approach to Informatics,” John Wiley and Sons, Inc., Hoboken, 2005. 